\title{
BASES PARA UN MODELO EDUCATIVO POR COMPETENCIAS EN TURISMO COMO HERRAMIENTA PARA EL DESARROLLO TURÍSTICO
}

Bases for an Educational Model Structured on Tourism Competencies as a Tool for Tourism Development

Yanira Loyola Tapia ${ }^{1}$

Claudia Oré Butler ${ }^{2}$

Recibido: mayo, 2018 // Aceptado: junio, 2018

\section{RESUMEN}

El continuo crecimiento del turismo en el Perú, así como las proyecciones positivas de esta actividad a nivel internacional y el desarrollo de nuevos mercados turísticos emergentes, obliga a los países a trabajar en nuevas estrategias educativas para formar un talento humano acorde con las necesidades de la industria del turismo y atender con eficiencia a un turista cada vez más exigente. Este documento se centrará en aquellos aspectos teóricos utilizados para el diseño y fortalecimiento de un modelo de educación superior en turismo, que toma en cuenta las tendencias en turismo en la educación superior. Se ha aplicado una metodología basada en un estudio de caso, con el propósito de presentar un modelo formativo en educación superior en turismo y sus implicancia. Para el desarrollo de las etapas relacionadas a la propuesta del modelo formativo se ha considerado la experiencia metodológica sugerida por la Organización Mundial del Turismo sobre capital humano. El modelo persigue la búsqueda de nuevos talentos capaces de cubrir las necesidades y exigencias del sector productivo. Este proceso educativo permitirá planificar y ejecutar una política educativa de turismo en el Perú y en otros países que se encuentran en el mismo camino de diseñar una educación superior para esta actividad. Se concluye que es necesario realizar ajustes en la educación superior para adaptarse constantemente a la forma como los conocimientos y contenidos deben ser impartidos, pues se trata de entornos cambiantes. Sobre la propuesta establecida, sirve de base y plataforma, pero debe ser renovada con cierta periodicidad según las circunstancias, sin dejar de lado la evaluación, medición y seguimiento del sistema educativo.

\footnotetext{
${ }^{1}$ Doctora en Educación, CENFOTUR. Líneas de Investigción: Calidad de la Educación y Desarrollo Regional, Trabajo Social, Administración. Dirección: Jirón Pedro Martinto 320, Distrito de Barranco, Lima - Perú. Teléfono: +51 3198000. E-mail: informacion@cenfotur.edu.pe

2 Maestra en Dirección y Planificación en Turismo, CENFOTUR. Líneas de Investigación: Educación Superior en Turismo, Desarrollo turístico, empleo en turismo. Dirección: Jirón Pedro Martinto 320, Distrito de Barranco, Lima - Perú. Teléfono: +51 3198000. E-mail: informacion@cenfotur.edu.pe
} 
Palabras clave: educación superior, competencias, transitabilidad, turismo, estudiantesprofesionales

ABSTRACT

The continuous growth of tourism in Peru, as well as the positive projections of this activity at the international level and the development of new emerging tourism markets, oblige countries to work on new educational strategies to form human talent according to the needs of the tourism industry and efficiently serve an increasingly demanding tourist. This document will focus on those theoretical aspects used for the design and strengthening of a model of higher education in tourism, which takes into account trends in tourism in higher education. A methodology based on a case study has been applied, with the purpose of presenting a training model in higher education in tourism and its implications. For the development of the stages related to the proposal of the formative model, the methodological experience suggested by the World Tourism Organization on human capital has been considered. The model pursues the search for new talents capable of covering the needs and demands of the productive sector. This educational process will allow for the planning and the execution of an educational policy in tourism in Peru and in other countries that are on the same path of designing a strategy of higher education for this activity. It is concluded that it is necessary to make adjustments in higher education to constantly adapt to the way knowledge and content should be imparted, since these are changing environments. The established proposal, serves as a base and a platform, but it must be renewed with certain periodicity, according to the circumstances, without neglecting the evaluation, measurement and monitoring of the educational system.

Key words: Higher Education, Competences, Transition Tourism, Student-Professionals

\section{INTRODUCCIÓN}

En una actividad en expansión global como el turismo, la formación de profesionales idóneos para el sector es clave para lograr la competitividad internacional, pero sobre todo es indispensable que la formación concuerde con las necesidades reales del mercado turístico de un país. Persiguiendo este objetivo, aquí se presentan las implicancias del diseño de un modelo formativo basado en competencias y su aplicabilidad en el Centro de Formación en Turismo (CENFOTUR), un organismo técnico especializado que se dedica hace 39 años a la educación, capacitación y perfeccionamiento del capital humano vinculado al sector turismo. 
Se desarrollarán aquellos aspectos teóricos analizados para el diseño y fortalecimiento del modelo de educación superior en turismo basado en competencias. La experiencia educativa de CENFOTUR será un punto de apoyo para mostrar la aplicación del mismo. Así, se podrán evidenciar algunos resultados basados en un aspecto clave de la formación de talento humano, como son las actividades en situación real de trabajo que se producen a través de las pasantías y/o prácticas profesionales. Por otro lado, se realiza un análisis de los ámbitos turístico, educativo y laboral del Perú, así como las propuestas de diferentes autores sobre el enfoque por competencias y su importancia para la formación de futuros profesionales.

Es importante mencionar que la trayectoria institucional del CENFOTUR ha permitido planificar y ejecutar una política educativa en turismo en el Perú, haciendo de esta casa de estudios un referente nacional de la educación superior en turismo.

Confiamos en que esta propuesta sirva de base para otras instituciones educativas del nivel superior de la región Latinoamérica que estén iniciándose en el camino de la formación de profesionales, o para aquellas que se encuentren en un proceso de modernización o mejoramiento de los planes de estudio del proceso formativo.

\section{MARCO TEÓRICO}

Antes de iniciar el planteamiento de un modelo educativo en turismo es necesario mencionar que en el Perú existe una política en turismo que ha impactado de forma positiva en el capital humano. Según la Ley General de Turismo (2009, artículo 47), el Estado ha propiciado “[...] el desarrollo y la mejora de las capacidades de los recursos humanos de mando básico (técnico-productivo), medio (técnico y profesionaltécnico) y superior (universitario y no-universitario) con la finalidad de ofrecer una mejor calidad del servicio al turista en el país” e impulsar el desarrollo turístico.

Esta política fija la ruta para desarrollar una educación superior en turismo planificada y en continuo perfeccionamiento. La actualización y mejora constante es una necesidad, debido al carácter cambiante del turismo, entre otros aspectos que se mencionarán posteriormente.

La educación en turismo en el Perú ha tomado gran importancia en los últimos años, no solamente por su crecimiento sostenido, sino porque 
los centros de estudio y, en general, el sector educación han evaluado la necesidad de incluir al turismo dentro de sus carreras profesionales. Esto se debe, principalmente, a que el turismo es uno de los sectores que más aporta al desarrollo económico del país y a la mejora de la calidad de vida. A pesar de este impulso renovado "existe escasa información específica [...]” (Regalado y Oré, 2009) con respecto a la educación superior para el turismo. Ello nos lleva a realizar un análisis basado en los vínculos entre la educación superior, el sector trabajo y el turismo, tomando en cuenta los requerimientos y necesidades de los empleadores, variables que permitirán plantear o reforzar un modelo educativo.

\section{ANÁLISIS DE ENTORNOS VINCULADOS}

Es necesario analizar tres sectores que son claves para proyectar un desarrollo del capital humano para el turismo. Estos son: educación, trabajo y turismo.

Ámbito turístico

En el Perú es visible un incremento de turistas debido -entre otros factores- a la estabilidad económica y social de las últimas décadas, a la apertura económica hacia los mercados internacionales y la política de promoción del turismo. Según la Superintendencia Nacional de Migraciones (2018), el crecimiento turístico se ve reflejado en el aumento del flujo de turistas desde el 2007, donde año a año se ha incrementado progresivamente, tal como se indica en la Figura 1. Al 2017, el Perú recibió cerca de 4.000.000 de turistas internacionales. 
Figura 1. Llegada de turistas internacionales entre los años 2007 y 2017, según la Superintendencia Nacional de Migraciones

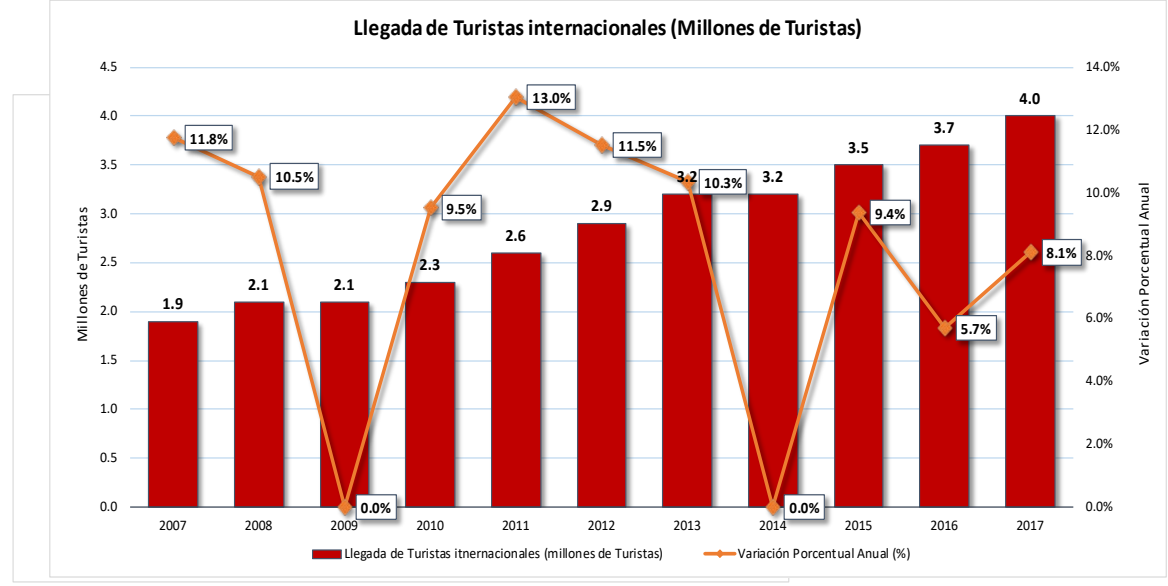

Fuente: Superintendencia Nacional de Migraciones/MINCETUR, 2018. Elaboración: MINCETUR-CENFOTUR

Teniendo en cuenta estas cifras desde el 2007 al 2017 se observa un aumento del 10.8\% del flujo de turistas y del 2016 al 2017 un $8.1 \%$ lo que indica un movimiento constante de visitantes.

Este dinamismo se expresa también en el crecimiento del flujo de turistas nacionales, en el aumento y diversificación geográfica de las inversiones nacionales y extranjeras en hoteles y restaurantes, y en el incremento del ingreso de divisas. Esto ha llevado a que algunas empresas peruanas se especialicen en el turismo de lujo, inversión que, a pesar de ser más cuantiosa, amplía el margen de ganancia.

En cuanto a los servicios turísticos, el sector hotelero es el de mayor crecimiento en el Perú.

Según el Ministerio de Comercio Exterior y Turismo ([Mincetur], 2016), existen cerca de 19. 000 hoteles clasificados en todo el país y en los próximos dos años ingresarán cerca de doce cadenas hoteleras (El Comercio, 2015), por lo que se avizora un buen desenvolvimiento de la oferta. Este panorama indica el incremento de mayores inversiones en hoteles de tres, cuatro y cinco estrellas, los cuales podrían llegar a superar los 700 millones de dólares. 
Según el Mincetur (2015), 4.800 nuevos establecimientos de hospedaje se integraron a la oferta turística del país entre el 2011 y 2015, pasando de 14.800 a 19.600 establecimientos, lo cual significó un incremento del $33 \%$. Asimismo, el número de habitaciones ofrecidas a los turistas creció en un 18 \%, al pasar de 208.000, en el 2011, a 246.000 en el 2015. Por otra parte, en los últimos años se aprecia la expansión de la oferta de hoteles, sobre todo en los de 4 y 5 estrellas, que presentan una tasa de crecimiento promedio anual de $10 \%$ y $5.8 \%$, respectivamente, durante el periodo 2010 al 2016 (Mincetur, 2016). Según la Sociedad de Hoteles del Perú, en el país operan 21 cadenas hoteleras, las cuales están concentradas especialmente en Lima. Según la Superintendencia Nacional de Migraciones, se proyecta que en el Perú se construyan 62 hoteles que demandarán una inversión de US\$ 1.141 millones durante el período 20172021 (Mincetur, 2015).

Es importante señalar que la oferta de servicios en el Perú está básicamente centrada en hoteles y, recientemente, en el rubro de restaurantes. Ello nos llevará a deducir que la formación de los futuros profesionales podría estar dirigida a preparar personas calificadas especialmente para estas actividades.

Por otro lado, la Dirección General de Investigación y Estudios sobre Turismo y Artesanía ([DGIETA], 2016) indicó que la actividad turística en el Perú emplea a cerca de 1.285.000 trabajadores, y gran parte de este número labora en el sector hotelero. La oferta hotelera se concentra en Lima, ciudad que tiende a convertirse en un destino para el desarrollo del turismo de convenciones o MICE (Meetings, Incentives, Conferencing, Exhibitions), lo que exige desarrollar una mayor oferta de servicios de calidad. Asimismo, el turista peruano ya planifica su viaje y recorre el país continuamente, movilizándose a las principales regiones. Un claro ejemplo de este incremento es que solo en el 2014 el total de viajes que realizaron fue de 4.48 millones (Promoción del Perú para la Exportación y el Turismo [PROMPERU], 2015). Esta situación a su vez genera la necesidad de mejorar los servicios a nivel nacional.

Esta mirada del turismo no puede estar desligada de los mercados, los cuales van cambiando rápidamente, especialmente los perfiles de los viajeros, por lo que es necesario formar profesionales capaces de adaptarse a estos cambios. Hoy en día la mayoría de ellos realiza sus transacciones de viaje de manera virtual. Las tendencias en turismo también marcan la 
pauta para realizar proyecciones a futuro sobre el desarrollo turístico nacional e internacional.

A nivel mundial, según Henley Centre HeadlightVision (s.f.):

Los viajeros del mañana demandarán niveles más altos de control, comodidad, seguridad y personalización, y la constante sofisticación de las tecnologías de la información permitirá a los proveedores satisfacer estas expectativas, transformando la experiencia del cliente desde la reserva hasta la recogida del equipaje (p. 4).

El autor también indica que otra tendencia es el incremento de los viajeros denominados “mayores activos”, que en el 2020 tendrán entre 50 y 75 años. No hay que dejar de considerar que también habrá un aumento de la población y que, por ello, será necesario una mayor información. Todo ello exige al futuro profesional en turismo adaptarse a los requerimientos de los clientes-turistas cada vez más exigentes. Del mismo modo, el panorama del turismo mundial muestra un turista cada vez más tecnológico y presenta destinos adaptados al cambio climático, mucho más temáticos e innovadores.

Ámbito educativo

La educación superior en turismo presenta una gran oferta de centros de estudio a nivel nacional, los cuales cuentan con un plan pedagógico muy heterogéneo, donde no necesariamente se consigue formar profesionales y técnicos con las competencias, habilidades y destrezas necesarias para que cierre algunas brechas el sector. A esto se suma que existe una carencia de información y estudios sobre la real situación de la educación superior en el sector turismo en el Perú donde, por ejemplo, se pueda visualizar si los futuros profesionales tendrán un puesto de trabajo al culminar sus estudios. Sin embargo, sí se cuenta con estudios vinculados a la educación superior en general, realizados por el Ministerio de Educación, que advierten sobre la necesidad de transformar la misma, conectando de forma más rápida al individuo y al sector productivo.

El panorama de la educación superior en el Perú se muestra aún con serias debilidades como, por ejemplo, la escasa articulación de 
competencias y unidades temáticas curriculares pertinentes para que el profesional pueda desenvolverse en un centro laboral.

Según información del proyecto Procalidad del Sistema Nacional de Evaluación, Acreditación y Certificación de la Calidad Educativa (Sineace) "la educación superior en el Perú atraviesa hoy por una fase de crecimiento económico y generación de empleo, sin embargo, el crecimiento no se ha producido pues hay un nexo entre este problema y la competitividad [...]”. (Procalidad, 2014, p. 37). Un aspecto importante para la competitividad es el elemento innovación, el cual debe ser tomado en cuenta en el diseño del nuevo modelo formativo. Es necesario considerarlo como un tema transversal a todas las carreras profesionales para turismo, pues sin innovación no hay competitividad; la idea es diferenciarse en el mercado.

Otro aspecto clave a tener en cuenta sobre la educación superior en el Perú es la vinculación educación-producción, que apunta a crear una educación superior de calidad para el desarrollo y la competitividad nacional (Consejo Nacional de Educación [CNE], 2005). Este enunciado exige analizar la educación superior y definir sus rumbos teniendo en cuenta la productividad y la formación de un potencial humano en distintos tipos de actividad, a nivel de diversas ocupaciones, desde las operativas hasta las gerenciales. Ello indica, además, que es imposible desvincular el trinomio educación-trabajo-producción, que es la fórmula que está siendo integrada al modelo educativo que plantea el CENFOTUR a través de su plan de estudio.

La Organización para la Cooperación y el Desarrollo Económicos ([OCDE], 2016) menciona dos aspectos clave que los países deben considerar a la hora de diseñar sistemas educativos: "Visualizar un aprendizaje para el empleo y alinear las ofertas educativas con las estrategias de crecimiento económico, teniendo en cuenta la visión país” (OCDE, 2016, p. 133) En el Perú es vital mantener un trabajo más articulado entre las instituciones de educación secundaria y los centros de educación técnico-productiva, o entre los institutos superiores tecnológicos. Para ello, las carreras deben adecuarse a la dinámica del mercado de la producción y servicios (CNE, 2005).

Este panorama da algunas luces sobre cómo se deben definir las líneas base para un modelo de formación en turismo. La participación del 
Estado es vital en la mejora del marco de políticas de la formación de capacidades laborales.

Regalado y Oré (2009) afirman que la formación profesional en turismo de las universidades e institutos superiores del Perú ha crecido vertiginosamente y sin control en los últimos cinco años. De acuerdo con investigaciones de las principales universidades, la oferta educativa en turismo está centralizada en Lima donde se encuentran más de veinte centros superiores que poseen una carrera o especialización universitaria en turismo. Aunque se trata del principal mercado, se debe mencionar que algunos de estos centros superiores son de baja calidad.

Basándonos en investigaciones propias, consideramos que muy pocas universidades han sido reactivas con respecto a este tema, y solo en algunos casos hubo una preocupación por actualizar los planes de estudio, mejorar los procesos y dar mayores oportunidades a los jóvenes. Algunas de ellas analizaron la necesidad de incluir al sector empresarial para elaborar sus mallas y abordar el estudio del turismo desde una visión multidisciplinaria, es decir, impartiendo conceptos desde diferentes disciplinas académicas, como la economía, la sociología o la contabilidad. Esta circunstancia ha llevado a que en la actualidad existan profesionales con competencias y conocimientos sobre desarrollo, planificación y comunicación, que tomen decisiones y que ejecuten planes y proyectos.

Por otra parte, desde el 2003, Educared mencionado en la publicación "La educación superior en turismo y las necesidades reales del mercado” ESAN (2009, p. 43) establece que hay una débil articulación entre los centros de educación superior y el desarrollo turístico nacional. Exceptuando a muy pocas instituciones indica que hay una escasa cultura de trabajo conjunto, lo que se refleja en diversos indicadores:

- Exceso de oferta de egresados en varias profesiones.

- Desvinculación entre los planes de desarrollo formativo y los planes de crecimiento empresarial.

Como menciona la UNESCO (1998), en el contexto actual de la educación es necesario propiciar el aprendizaje permanente y la construcción de las competencias adecuadas para contribuir al desarrollo cultural, social y económico de la sociedad. Para cumplir con ello, la educación superior debe impulsar la generación de nuevos conocimientos -por ejemplo, mediante la investigación constante-, entrenar personas altamente calificadas, proporcionar servicios a la sociedad y fomentar la 
crítica social. Así, los gobiernos y sectores productivos que impulsan políticas que promueven el crecimiento económico deben realizar esfuerzos por mejorar la formación de capacidades de la fuerza laboral (Banco Mundial, 2010). Este impacto positivo de crecimiento sostenido puede mejorarse promoviendo la formación de capacidades y un mejor emparejamiento de trabajadores con empleos idóneos, mediante programas bien diseñados que ayuden a la economía a adaptarse, como es el caso del turismo.

La presencia del Estado es fundamental en la mejora de las políticas de la formación de capacidades laborales que brinden el marco que guíe a las instituciones educativas en el modelo de educación y capacitación relevante de alta calidad, regulando los mercados laborales para que funcionen según las reglas económicas y donde los trabajadores puedan convertir sus capacidades en empleo productivo.

A pesar de las dificultades, la indagación y la experiencia de centros superiores en turismo, como el CENFOTUR, son indispensables para definir un modelo educativo con visión de futuro que cree y se transforme a lo que realmente requiere el sector turismo en el Perú.

\section{Ámbito laboral}

Sobre las demandas del entorno económico productivo, existe una exigencia cada vez más continua por parte del empresariado con respecto a la renovación constante de los diseños curriculares como principal medida, al igual que de los planes institucionales o proyectos educativos de las entidades de educación superior. Es necesario tomar en cuenta las necesidades del desarrollo regional y nacional, poniendo especial énfasis en la formación de líderes éticos y con sentido de responsabilidad social. Para esbozar un modelo educativo se debe tener en cuenta las necesidades y demandas de los empresarios, no solamente a nivel de Lima como principal mercado turístico del Perú, sino en los principales mercados turísticos del país como son Arequipa, Cusco, Ica, Puno, Madre de Dios e Iquitos.

Si bien las demandas para el sector turismo son medianamente conocidas por las instituciones educativas en el Perú, la comunicación entre la academia y los empresarios es escasa. Según el Instituto Nacional de Estadística e Informática ([INEI], 2015) el sector turismo a nivel de 
productividad en el trabajo y de calidad del empleo respecto a otros sectores de la economía peruana, presenta un $51.6 \%$ de trabajadores que posee educación secundaria completa y solo el 14.6 \% cuenta con educación superior no universitaria. Estos bajos niveles de educación constituyen un desafío de política pública si se quiere mejorar la productividad y la competitividad del turismo. De acuerdo al estudio del Ministerio de Trabajo y Promoción del Empleo ([MTPE], 2013), una condición para alcanzar mayores niveles de competitividad en la actividad turística implica mejorar la productividad de sus trabajadores.

Un aspecto importante del ámbito laboral actual en el sector turismo es que el $47.5 \%$ de los trabajadores de la actividad turística trabaja por cuenta propia, es decir, se desempeña como empleador o como independiente en condición de autoempleo (MTPE, 2013). En la Encuesta Nacional de Hogares 2015 (INEI, 2015) se menciona que la actividad turística emplea a personas jóvenes: el $25.7 \%$ tiene entre 18 y 29 años, menos de 24 años de edad y un 32.8\% tiene entre 30 y 39 años; sin embargo, valga aclarar que esta es una característica común en todos los sectores de la economía peruana. En cuanto al rubro de restaurantes, el empleo formal registrado durante el año 2011 ascendió a 144.639 trabajadores (MTPE, 2013). Esta cifra no incluye a los trabajadores informales.

Un aspecto a tomar en cuenta y que luego será comprobado en los resultados de aplicación del modelo por competencias, es que el empleo en el sector turismo tiene rostro de mujer, tres de cada cuatro trabajadores son mujeres y trabajan básicamente en el hotelería (INEI, 2015)

Es necesario tomar en cuenta esta información para elaborar modelos educativos considerando que existen aspectos por reforzar para conseguir la competitividad del sector. Para ello, se podrían diseñar e implementar sistemas de capacitación que incentiven la productividad operativa en la actividad turística.

\section{BASES TEÓRICAS PARA UN MODELO EDUCATIVO POR COMPETENCIAS}

La formación de profesionales para el turismo requiere de un sistema educativo flexible y dinámico que responda con eficiencia a las exigencias de la industria del turismo. De acuerdo al análisis precedente existen aspectos que deben ser integrados y contrastados con aquellos 
lineamientos y/o bases teóricas que sustentan el diseño de un enfoque educativo por competencias. Si bien el modelo por competencias no es nuevo en el Perú ni en el mundo, pues existen evidencias de su aplicación, es importante presentar algunas propuestas y planteamientos teóricos sobre este enfoque, así como los beneficios de la aplicación y su posterior adaptación de los procesos tradicionales de enseñanza a un modelo por competencias y como este debe ir adecuándose al crecimiento del sector productivo.

Existe una amplia bibliografía para definir las competencias, pero en este caso creemos pertinente mencionar la que propone la OCDE, la cual indica que es "un grupo de conocimientos, atributos y capacidades que pueden aprenderse y que posibilitan a los individuos realizar una actividad o tarea de forma exitosa y consistente, y pueden construirse y extenderse a través del aprendizaje” (2016, p. 19).

Barrales (2012) comparte la experiencia que ha tenido la Universidad Veracruzana en la promoción e implementación del enfoque educativo basado en competencias. El proyecto estuvo dirigido a sistematizar la práctica docente a partir de la elaboración, aplicación y evaluación de un diseño instruccional para cada una de las experiencias educativas (asignaturas) que se imparten al interior de la universidad. La autora revisa diversos conceptos como competencia, tipos, enfoques, metodología y evaluación por competencias. De este documento extraemos un concepto concreto y esclarecedor de lo que es una competencia y que expuso Kofi Annan, Secretario de la ONU, en Asamblea General (citado en Barrales, 2012) “competencia es la combinación de habilidades, atributos y comportamientos que están directamente relacionados con un desempeño exitoso en el trabajo” (p. 26). Esta combinación es la que debe tener todo profesional al dejar las aulas y enfrentarse al mundo laboral, pero ello dependerá del diseño de planes de estudio y del dominio que tengan docentes en aplicar con eficacia un modelo educativo por competencias.

La experiencia de la Universidad Veracruzana mencionada por Barrales (2012) sobre la implementación de enfoque educativo basado en competencias, confirmó algunos retos y aciertos, de los cuales resaltamos:

1. La resistencia académica en cuanto a cambiar de paradigma: que tiene que ver con romper los esquemas tradicionales de enseñanza para dar paso a la aplicación de un sistema por competencias. Esto 
significa no solo adaptarse institucionalmente, sino introducir al docente en este nuevo sistema, reacción que de alguna manera se ha observado en el proceso de cambio de los planes de estudio del CENFOTUR que se verá posteriormente.

2) "Incrementar la participación en un sistema de evaluación común interinstitucional para profesionales, que garantice el intercambio de estudiantes con otras instituciones educativas" Barrales (2012, p. 29). Es decir, buscar que un profesional formado con un enfoque por competencias de una institución educativa lo sea igualmente para todas las instituciones educativas que promuevan las mismas carreras. Esto implica que no debe importar de qué universidad sea, pero sí que cumpla con los requerimientos académicos, conocimientos, habilidades y tenga el mismo desempeño.

A manera de conclusión la autora indica: Las instituciones de educación superior deben revisar "los objetivos de aprendizaje, actividades, estrategias de evaluación y calificación, a fin de garantizar la coherencia, confiabilidad y validez de los resultados”. Barrales (2012, p. 38). Estos resultados dan una pauta sobre aquellos aspectos a tomar en cuenta para el diseño de un modelo educativo adaptado al sector turismo.

Por otro lado, Argudín (2005) indica que “es necesario propiciar el aprendizaje permanente y la construcción de las competencias adecuadas para contribuir al desarrollo cultural, social y económico de la Sociedad de la Información” (p. 2). Del mismo modo, un tema abordado por la autora es que las competencias comprenden una nueva dimensión que va más allá de las habilidades o destrezas. Por ejemplo, dos personas pueden haber desarrollado sus habilidades al mismo nivel, pero eso no significa que van a construir un producto con la misma calidad y excelencia. La competencia implica algo más que se expresa en el desempeño.

El modelo de educación basado en competencias se puede definir como un proceso con una intencionalidad, es decir, una dirección, mediante la cual se pretende lograr un conjunto de cambios de actitud y conducta del individuo en relación al mundo que lo rodea y a los demás miembros de la sociedad.

Un análisis más exhaustivo realiza Le Boterf (2000) sobre lo que significa competencia, y explica cómo el concepto ha ido evolucionando desde la concepción de Taylor que tradicionalmente implicaba que un 
trabajador cumpliera una tarea específica, así como seguir las instrucciones necesarias, y donde el saber-hacer era lo más importante a una competencia que es más que saber-hacer, en el que es necesario demostrar iniciativa, ser capaces de adaptarse a coyunturas y tener actitud. En este sentido, la competencia la define más en términos de saber-actuar que de saber-hacer, todo ello debido a los cambios que están ocurriendo en las empresas y la forma en cómo se organiza el trabajo.

A nivel internacional algunos países vienen aplicando modelos educativos por competencias o similares con una fuerte vinculación con el mercado laboral. Este enfoque se observa en países de Latinoamérica, Europa y Asia. Un caso muy interesante es el de Colombia país que aplica una educación vinculada al trabajo a través del Servicio Nacional de Aprendizaje. Ministerio de Educación de Colombia (2016). Este busca mejorar las capacidades y competencias del recurso humano a nivel técnico, tecnológico y de formación profesional. Otro país que viene desarrollando una formación por competencias es Corea país que forma recurso humano para las industrias, cubriendo las necesidades específicas de cada una de ellas.

La propuesta teórica expresada nos lleva a plantear los siguientes objetivos concretos. Posteriormente, se explicarán sus principios metodológicos.

Objetivos Principales

- Proponer y analizar un modelo formativo en turismo adaptado a las exigencias del mercado laboral-productivo.

- Difundir un modelo de educación superior para el sector turismo en Latinoamérica como un aporte base para la discusión de la academia.

Objetivos Específicos

- Acreditar las competencias de manera progresiva con el propósito de logar un horizonte académico y laboral.

- Impulsar la vinculación constante entre los planes de estudio y el mercado laboral del sector turismo con miras al perfeccionamiento del capital humano. 


\section{METODOLOGÍA}

Se ha aplicado una metodología basada en un estudio de caso, con el propósito de presentar un modelo formativo en educación superior en turismo y sus implicancias. Si bien resulta ser una investigación teórica, se indican los procesos por los cuales se está validando el modelo propuesto. Se ha realizado también un levantamiento de información sobre los entornos vinculantes, realizando un análisis de los mismos.

Para el desarrollo de las etapas relacionadas a la propuesta del modelo formativo se ha considerado la experiencia metodológica sugerida por la Organización Mundial del Turismo sobre capital humano, que permite definir la situación actual en el país e identificar las brechas entre la educación y el mercado.

\section{MODELO FORMATIVO POR COMPETENCIAS: PROPUESTA, IMPLICANCIAS Y RESULTADOS}

Para diseñar un modelo formativo por competencias para ser validado en el espacio educativo del CENFOTUR, se ha realizado un análisis comparativo del enfoque del modelo formativo basado en objetivos, tal como se muestra en la Tabla 1 sobre diferencias entre el modelo formativo por objetivos y el modelo por competencias.

Dentro de este análisis se ha descifrado que el enfoque tradicional por objetivos está basado meramente en contenidos por cada asignatura que da como resultado un modelo rígido que enfatiza la enseñanza de asignaturas establecidas en los syllabus. Así se acentúa el conocimiento y comprensión de cada una de ellas, pero se deja de lado la interrelación e interdisciplinariedad de las materias y la transferencia de las mismas.

Teniendo en cuenta el problema, se observó que este modelo tenía debilidades, ya que, debido a su rigidez, es inflexible a los cambios especialmente teniendo en cuenta que el turismo está en constante evolución. Por ello, se determinó la necesidad de aplicar un enfoque por competencias. 
Tabla 1. Diferencias entre el modelo por objetivos y el modelo por competencias

\begin{tabular}{|c|c|}
\hline Modelo formativo por objetivos & Modelo formativo por competencias \\
\hline $\begin{array}{lcrr}\text { Enfoque } & \text { tradicional basado } & \text { en } \\
\text { contenidos y enseñanza de } & \text { las } \\
\text { asignaturas establecidas. } & & \end{array}$ & $\begin{array}{l}\text { Enfoque basado en el desempeño y } \\
\text { transferencia, donde el protagonista es el } \\
\text { estudiante. Respuesta colegiada con el } \\
\text { sector productivo y levantamiento de } \\
\text { demandas laborales. }\end{array}$ \\
\hline $\begin{array}{l}\text { Rígido, no preparado de manera } \\
\text { inmediata para los cambios en el sector. }\end{array}$ & $\begin{array}{l}\text { Flexibilidad, planes o mallas curriculares } \\
\text { acordes a las necesidades y demandas del } \\
\text { sector productivo. }\end{array}$ \\
\hline $\begin{array}{l}\text { Enfatiza la enseñanza de cada asignatura } \\
\text { establecida en los syllabus. }\end{array}$ & $\begin{array}{l}\text { Syllabus busca poner énfasis en la } \\
\text { transferencia. }\end{array}$ \\
\hline Inflexible a los cambios. & $\begin{array}{l}\text { Movilización y articulación para transitar } \\
\text { de un nivel de formación a otro. }\end{array}$ \\
\hline $\begin{array}{l}\text { Deja de lado la interrelación con otros } \\
\text { sectores vinculados al turismo. }\end{array}$ & $\begin{array}{l}\text { Teórica y empíricamente conectado con } \\
\text { los sectores trabajo y educación. }\end{array}$ \\
\hline No realiza implementación & $\begin{array}{l}\text { Revisión y adecuación permanente } \\
\text { después de la implementación. }\end{array}$ \\
\hline
\end{tabular}

Elaboración propia: Cenfotur, 2016

Estamos convencidos de que el modelo por objetivos debe ser transformado para convertirse en uno por competencias. Los beneficios son diversos y se observarían sus resultados, paulatinamente, en las distintas escalas del sector del turismo nacional. Este modelo permitiría transitabilidad, lo que ofrecería la movilización necesaria para que los estudiantes puedan transitar de un nivel de formación a otro a través de la articulación. Esto incrementaría las competencias desde el nivel técnico al profesional. Al responder de manera oportuna y pertinente con mallas y contenidos curriculares que promueven la movilización educativa, se logrará brindar accesibilidad a la educación técnica superior y formar personas competentes y de alta productividad, que den respuesta a las demandas del sector.

La formación brindada a partir de una pertinencia curricular estructurada, que considere la realidad laboral, debe ofrecer al profesional titulado y a los practicantes preprofesionales la capacidad para interactuar con el entorno, a partir de las estructuras organizacionales y sus procesos modificadores. En el caso del Perú, es necesario contar no solamente con profesionales para cubrir puestos vinculados a los servicios turísticos, sino 
también para cubrir cargos directivos y técnicos relacionados con la planificación y la gestión en turismo, necesario en las regiones turísticas del Perú.

Dentro de sus competencias, CENFOTUR prepara actualmente jóvenes para afrontar los desafíos de la industria del turismo. Es parte de su competencia brindar un modelo formativo basado en un enfoque más moderno y flexible, acorde con las necesidades del mundo del trabajo. Consideramos que el modelo por competencias cubriría estas necesidades. Sin embargo, dada la coyuntura de cambio constante en la que vivimos, repasada páginas atrás, la propuesta pedagógica debe ser constantemente revisada y adecuada a las nuevas necesidades de la industria turística.

El modelo por competencias, asentado en las bases teóricas ya mencionadas, le permite al estudiante de nivel técnico obtener el título profesional, una vez aprobados los módulos y los requisitos de dicho nivel. Las características de este modelo están siendo sustentadas por conceptos impulsados en el ámbito académico peruano como la pertinencia curricular, ya mencionada, la productividad del currículo que considera las salidas intermedias al mercado laboral y la certificación progresiva, tal como puede evidenciarse en la Figura 2.

Figura 2. Modelo formativo. Convalidación de cursos hasta llegar a la profesionalización

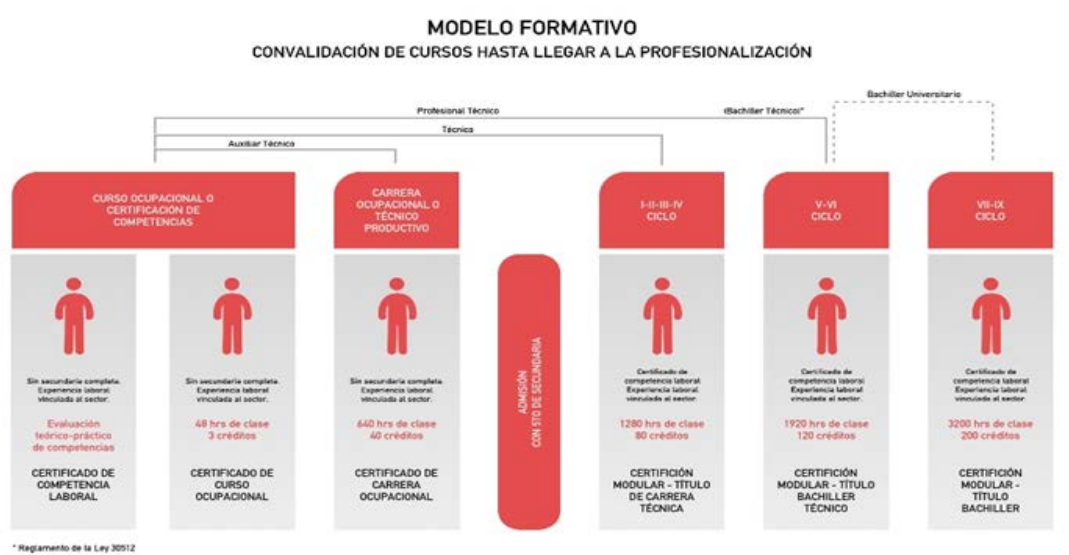

Actualmente el modelo se está aplicando en el CENFOTUR y se realiza una difusión del mismo a través de acuerdos y convenios con otras 
instituciones educativas públicas y privadas donde se transmite y divulga la experiencia del modelo formativo. Asimismo, a través del Ministerio de Educación se realiza una promoción del mismo a través de sus programas sectoriales como el Programa Nacional de Becas y Crédito EducativoPRONABEC.

\section{ETAPAS DEL DISEÑO DEL MODELO POR COMPETENCIAS}

Para el diseño de un modelo formativo que se relacione con el mundo laboral, se proponen las siguientes etapas.

Primera etapa

Articulación entre la oferta educativa y la demanda laboral

Con la intención de buscar la articulación entre la oferta educativa de la educación superior y la demanda laboral, se definió que el nuevo Plan de Estudios debería ser trabajado con representantes del sector empresarial privado, los gremios del sector turismo, exalumnos y docentes. Para ello se determinó como estrategia la realización de mesas de trabajo o workshops donde intervinieron profesionales especialistas en cada tema, de las instituciones antes referidas, y que se muestra en la Figura 3.

Figura 3. Estrategia utilizada: realización de mesas de trabajo.

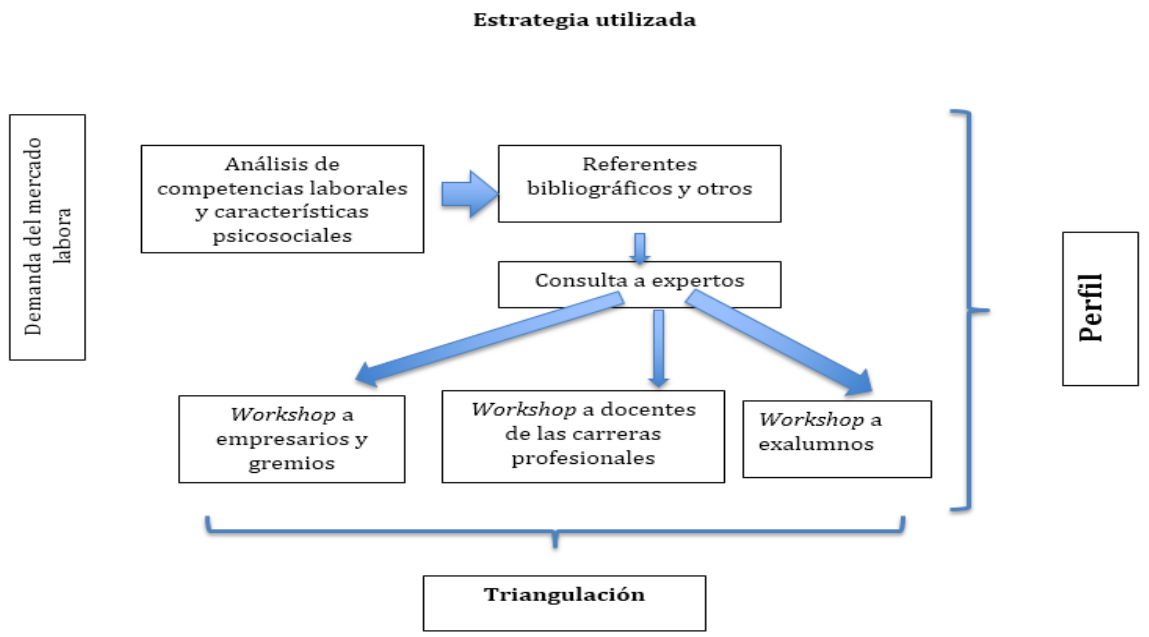

Fuente: elaboración propia 
Los workshops realizados tuvieron el siguiente propósito:

- Identificar la carrera, especialidades y opciones ocupacionales que actualmente son necesarias para el desarrollo del sector turismo del país.

- Identificar nuevas opciones ocupacionales y/o familias profesionales que deben ofrecerse.

- Evaluar la pertinencia de las carreras profesionales que actualmente se brindan en el CENFOTUR.

- Identificar y sugerir los ajustes necesarios a los perfiles profesionales de las carreras que se deben seguir ofreciendo, con base en la práctica y una visión prospectiva.

- Recopilación de información de diversas fuentes: levantamiento del perfil de egreso, validación del perfil de egreso, integración de ajustes, y validación por parte de diferentes actores, donde se establece la triangulación entre gremios y empresa privada, exalumnos y docentes.

- Socialización del perfil de egreso.

Esta estrategia incluye seis pasos fundamentales:

1. Reconocer las características principales de las exigencias y demandas del mercado laboral, identificando sus procesos clave: competencias generales, específicas y profesionales. Estos últimos son aquellos que definen los módulos principales que deberá contener el proceso formativo.

2. Identificar las distintas capacidades y actividades que involucra cada nivel de competencias, es decir, las diferentes subfunciones que el trabajador debe desempeñar en el contexto laboral. En términos del proceso formativo, este paso sirve para identificar las posibles asignaturas que cubrirán esos desempeños.

3. Definir contenidos específicos para el logro de cada actividad, para lo cual es necesario revisar los criterios de desempeño y las evidencias de conocimientos adecuados. En el proceso formativo esto se traduce en la pregunta: ¿qué contenidos necesita aprender el estudiante para lograr dicha actividad?

4. Identificar los conocimientos y el prerrequisito o conocimientos previos que puedan dar soporte al aprendizaje específico. 
5. Definición final de las asignaturas a través de la organización de conocimientos específicos. Es el momento en el que además se define la secuencia curricular.

6. Finalmente, la definición de la carga horaria y/o créditos de acuerdo al nivel de competencia y al perfil requerido para la carrera profesional. Con ello se determina el tiempo semanal necesario que demanda el desarrollo de los contenidos de cada asignatura, así como los créditos, con el fin de convalidar y/o homologar la formación continua de los estudiantes en su proceso de mejora.

Estos pasos son revisados y validados de manera independiente, con el fin de dar confiabilidad, validez y pertinencia al resultado en el nivel de plan de estudios.

Segunda etapa

Movilización y articulación entre los niveles educativos

La propuesta que se viene implementando en CENFOTUR, tiene como característica brindar una oferta educativa basada en competencias y organizada de manera curricular en módulos que permiten a los estudiantes avanzar de manera progresiva en la adquisición de conocimientos y niveles de competencia cada vez más amplios.

El aprendizaje no se comprende de manera lineal, sino más bien ascendente, siempre con la retroalimentación progresiva. En ese sentido, los planes de estudio de una carrera técnico-productiva, técnica y profesional deben responder al logro de un conjunto de competencias vinculadas al mercado laboral con puestos de trabajo articulados, que a su vez den lugar a una ocupación. Es por ello que este modelo es inclusivo, pues responde a una formación continua que permita la movilización y articulación entre los niveles educativos.

En consecuencia, para el caso del CENFOTUR, la organización curricular ha considerado la cantidad de horas y créditos que permitan la convalidación en cada uno de los niveles, con la finalidad de que los estudiantes puedan transitar de un nivel de formación a otro. Esto permite incrementar las competencias dentro de la institución desde el nivel técnico-productivo, técnico y profesional. 
Las características de este modelo están siendo sustentadas por conceptos impulsados en el ámbito académico peruano, donde se evalúan aspectos como la pertinencia curricular, la cual permite analizar temas como la capacidad del currículo para interactuar con el entorno, a partir de las estructuras organizacionales y sus procesos modificadores o la productividad que considera las salidas intermedias al mercado laboral y la certificación progresiva. Con ello, el currículo se vincula con la empleabilidad, pues se incluyen cursos que preparan al estudiante para el mercado laboral. Asimismo, se busca desarrollar el emprendimiento, que es un eje transversal en el modelo.

Tercera etapa

Correlación del modelo formativo y el marco de cualificaciones

La correlación del modelo formativo por competencias, además de lograr la movilización educativa, permite la transitabilidad del mundo laboral a la enseñanza formal, es decir, da pie para diseñar y construir un marco de cualificaciones para el sector turismo. Para ello, debe estar conectado con los sectores trabajo y educación de cada nación, y contar con empleadores y trabajadores en su elaboración. La participación de estos actores favorecerá el progreso profesional y promoverá la consideración social de las cualificaciones, debido a que una persona que presta servicios en el sector turismo puede acceder a una certificación de su competencia laboral en un determinado perfil ocupacional, validando así su experiencia en el rubro, siempre y cuando, claro está, cumpla con los requisitos formativos de las diferentes carreras técnico-productiva, técnica y/o profesional.

El sector requiere de personas altamente calificadas, con una formación integral y con capacidad de formarse y actualizarse a lo largo de su vida, logrando progresivamente mejores niveles formativos, creando conocimientos aplicados y soluciones, así como ser ciudadanos constructivos de una sociedad más justa y competitiva. En ese sentido, CENFOTUR es una pieza clave en la empleabilidad de los ciudadanos y en la mejora permanente de la competitividad del aparato productivo del sector turismo, que es un eje económico central y transversal del país.

Con respecto a estas tres etapas, se ha cumplido con ejecutar la primera y segunda etapa junto al sector productivo y la adecuación del plan 
de estudios. Así, en estos últimos tres años, ha comenzado a rendir sus frutos.

\section{RESULTADOS DE LA APLICACIÓN DEL MODELO FORMATIVO}

La propuesta presentada sobre un modelo formativo acorde con las exigencias de los mercados turísticos continúa siendo implementada en el espacio educativo del CENFOTUR. El modelo considera tres componentes curriculares: competencias técnico específicas, competencias para la empleabilidad y experiencias formativas en situación real de trabajo. En relación a la propuesta formativa que permite la transitabilidad de los alumnos de un nivel de formación a otro, el estudiante ha ido incrementando sus competencias dentro de la Institución. Esto ha permitido obtener algunos resultados vinculados a cada etapa del diseño arriba mencionado.

\section{Primera etapa}

En esta etapa se ha desarrollado el plan de estudios diseñado de acuerdo a las necesidades y demandas del mercado laboral. Desde el 2014 a la fecha, CENFOTUR viene implementado el modelo de formación por competencias, el cual cuenta con un esquema gradual de certificaciones modulares dado por las competencias obtenidas de los estudiantes al culminar de manera satisfactoria dos ciclos académicos. También ello está ligado al desarrollo de competencias profesionales, que permiten obtener oportunidades de trabajo y responder con calidad a los desafíos del mercado laboral actual. Ello se ha logrado vinculando a los estudiantes mediante pasantías que no es más que conectar al estudiante en situaciones reales de trabajo.

En este punto uno de los resultados obtenidos son las experiencias formativas que han tenido los estudiantes. Cada dos ciclos académicos los estudiantes van adquiriendo competencias de manera progresiva, las cuales son puestas a prueba en las pasantías iniciales, luego de estos ciclos el estudiante continúa realizando pasantías hasta la culminación de su carrera. 
Este proceso ha permitido obtener algunos resultados y hallazgos de interés tanto para la institución como para el sector turístico peruano. Los resultados refieren a información sobre el perfil de los jóvenes que se van insertando al mercado laboral. Aspectos como el género, un tema de gran relevancia a nivel social y que nos dice que el turismo en el Perú tiene rostro de mujer, así como las edades, y las preferencias de los mismos por un área específica de trabajo, son algunos de los resultados que se han ido obteniendo del proceso de aplicación del modelo educativo.

En la Tabla 2 se muestra que, en el año 2016, 123 estudiantes han realizado pasantías en empresas del sector, mientras que 19 estudiantes ya están laborando formalmente en una empresa, sin haber culminado aún sus estudios. Esto indica que existe una dinámica de empleabilidad de los estudiantes que han sido formados bajo el enfoque por competencia y favoreciendo al sector productivo.

Tabla 2. Inserción y vinculación de estudiantes y egresados, 2016.

\begin{tabular}{|l|c|c|}
\hline Inserción y vinculación & Estudiantes & Egresados \\
\hline Pasantías & 123 & 3 \\
\hline Administración Hotelera & 32 & 1 \\
\hline Administración Turística & 31 & 1 \\
\hline Guía Oficial de Turismo & 9 & \\
\hline Carrera Técnica de Cocina Peruana & 22 & 1 \\
\hline Prácticas Profesionales & 6 & 5 \\
\hline Administración Hotelera & 9 & 2 \\
\hline Administración Turística & 1 & \\
\hline Guía Oficial de Turismo & 1 & \\
\hline Guía Oficial de Turismo Virtual & 4 & \\
\hline Counter de aviación, turismo y agente de tráfico & 1 & \\
\hline Bar manager & 19 & 32 \\
\hline Colocaciones en Mercado Laboral & 4 & 29 \\
\hline Administración Hotelera & 9 & 1 \\
\hline Administración Turística & 1 & 4 \\
\hline Guía Oficial de Turismo & 1 & \\
\hline Carrera Técnica de Cocina Peruana & 4 & \\
\hline Cursos de Extensión Educativa & 164 & \\
\hline TOTAL & & \\
\hline
\end{tabular}

Fuente: Cenfotur, 2016 
Las colocaciones laborales son también el resultado de la aplicación del modelo formativo por competencias. Las empresas del sector turismo en el Perú demandan egresados con competencias y habilidades específicas, es así que se articulan las necesidades laborales con los perfiles de los estudiantes y egresados. En el 2016, se han colocado 82 egresados.

Asimismo, un aspecto interesante es que durante el proceso de inserción laboral en el mercado turístico existe un gran número de estudiantes que prefieren realizar sus pasantías en algún tipo de empresa en específico. Si observamos la Tabla 3, existe un grupo significativo de estudiantes que optan por trabajar en agencias de viajes y hoteles seguido por el área de restaurante.

Tabla 3. Tipo de empresa.

\begin{tabular}{|l|c|}
\hline Condición laboral - pasantías & Alumnos \\
\hline Tipo de empresa & 32 \\
\hline Agencia de Viajes & 5 \\
\hline AAVV & 1 \\
\hline Club Campestre & 2 \\
\hline Complejo arqueológico & 2 \\
\hline Educación & 44 \\
\hline Hotel & 3 \\
\hline Museo de Sitio & 40 \\
\hline Restaurante & 1 \\
\hline Restaurante y Bar & 130 \\
\hline Total & \\
\hline
\end{tabular}

Fuente: Cenfotur, 2016

Y como se indicó líneas arriba, en el caso de la variable género, como se observa en el cuadro superior, un $68 \%$ de los futuros profesionales de turismo son mujeres y se desenvuelven especialmente en hotelería, en cocina peruana y en administración turística. Un punto también de gran interés no solo para el sector turismo, sino para plantear políticas para la educación superior en turismo es que la fuerza laboral para este sector tiende a ser muy joven. Por ejemplo, el grupo de futuros profesionales pasantes más representativo tienen 24 y 25 años mientras que los de 22 años representan un 16\%, tal como se indica en la Tabla 4. 
Tabla 4. Condición laboral de las pasantías.

\begin{tabular}{|l|c|c|c|}
\hline Género & Femenino & Masculino & Total general \\
\hline Administración hotelera & 28 & 7 & 35 \\
\hline Administración hotelera & 1 & & 1 \\
\hline Administración turística & 30 & 5 & 35 \\
\hline Carrera de Técnica de cocina Peruana & & 1 & 1 \\
\hline Cocina peruana & 27 & 25 & 52 \\
\hline Guía Oficial de Turismo & 7 & 5 & 12 \\
\hline TOTAL & 93 & 43 & 136 \\
\hline$\%$ & $68 \%$ & $43 \%$ & 136 \\
\hline & & & \\
\hline
\end{tabular}

Fuente: Cenfotur, 2016

\begin{tabular}{|c|c|c|}
\hline Condición laboral de las pasantías. & Alumnos & $\%$ \\
\hline EDAD & & \\
\hline 17 & 5 & $4 \%$ \\
\hline 18 & 24 & $18 \%$ \\
\hline 19 & 22 & $16 \%$ \\
\hline 20 & 25 & $18 \%$ \\
\hline 21 & 19 & $14 \%$ \\
\hline 22 & 14 & $10 \%$ \\
\hline 23 & 12 & $9 \%$ \\
\hline 24 & 4 & $3 \%$ \\
\hline 25 & 4 & $3 \%$ \\
\hline 26 & 2 & $1 \%$ \\
\hline 27 & 4 & $3 \%$ \\
\hline 29 & 1 & $1 \%$ \\
\hline Total general & 136 & \\
\hline
\end{tabular}

Fuente: Cenfotur, 2016

Segunda etapa

Como ya se ha mencionado anteriormente, el modelo educativo responde a una formación continua y permite la movilización y articulación entre los niveles educativos, favoreciendo la convalidación en cada uno de los niveles.

En esta etapa, la cual se encuentra en pleno proceso de análisis, se están estudiando la capacidad del currículo para interactuar con el entorno, donde se consideran las salidas intermedias al mercado laboral, como son las prácticas preprofesionales y las pasantías. Las pasantías representan 
una acción clave para el cumplimiento del proceso de formación basado en competencias.

La convalidación en cada uno de los niveles se está analizando teniendo en cuenta el tránsito que viene realizando un primer grupo de estudiantes desde el nivel ocupacional hasta el nivel profesional y cuáles son las competencias adquiridas en cada etapa (ver Figura 4).

Se ha considerado el análisis de la transitabilidad entre:

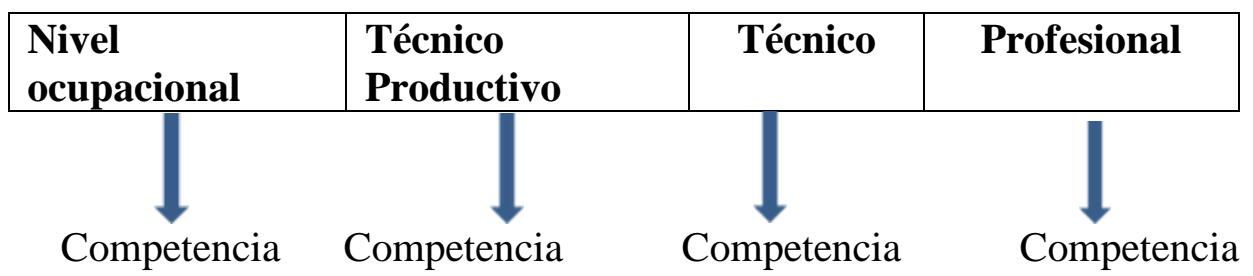

Figura 4. Análisis de transitabilidad.

Tercera etapa

La tercera etapa tiene que ver con el proceso del marco de cualificaciones para el sector turismo con participación del Ministerio de Trabajo y Promoción del Empleo y el Ministerio de Educación. Este proceso se encuentra en etapa de diseño, para luego construir las bases del mismo con la participación de empleadores y trabajadores del sector, ello facilitará el progreso profesional y la mejora de estándares en el sector turismo.

\section{CONCLUSIONES}

El análisis de la educación superior en turismo en el Perú es un tema de gran importancia en los espacios académicos y públicos; sin embargo, paradójicamente, es poco estudiado. El crecimiento del turismo en el país ha propiciado que se inicie una gran corriente de evaluación sobre el tema, especialmente de profesionales vinculados a la educación superior, a la economía y al trabajo, sin dejar de lado a la academia. En este marco, la educación superior en turismo toma relevancia, teniendo en cuenta que, si no existe un potencial humano idóneo para satisfacer las 
necesidades de los turistas y de las actividades turísticas, no es posible ser competitivos y convertirnos en un destino de calidad.

En esta línea, los centros de educación en turismo son claves para preparar profesionales para los distintos puestos de trabajo, con habilidades, destrezas y capacidad de adaptación. Es por ello que se ha propuesto un modelo educativo que puede ser aplicado a cualquier mercado turístico de Latinoamérica que busca la excelencia basada en el talento de profesionales idóneos.

De acuerdo a los hallazgos encontrados y a los procesos educativos ejecutados en CENFOTUR sobre el modelo educativo por competencias, es clave tener en cuenta tres temas: el crecimiento del turismo y los cambios vertiginosos que conlleva, el cambio climático y la era digital. Estos temas obligan a realizar ajustes en la educación superior para adaptarse constantemente a la forma como los conocimientos y contenidos deben ser impartidos, pues se trata de entornos cambiantes. Así, la propuesta establecida debe servir de base y plataforma, pero debe ser renovada con cierta periodicidad según las circunstancias, sin dejar de lado la evaluación, medición y seguimiento del sistema educativo.

El mercado laboral -y por tanto la empresa privada- junto al sector productivo son la pieza fundamental para tomar el pulso a las necesidades reales de profesionales y técnicos para atender a los turistas-clientes. Los actores del mercado son los interlocutores válidos para conocer cuáles son las habilidades, conocimientos y destrezas que debe tener un profesional del sector turismo. Es vital aplicar una consulta previa y realizar un análisis continuo del mercado laboral y cuáles son las debilidades actuales. Esta acción ayudará a sentar las bases para los contenidos curriculares de los centros de formación en turismo.

Si bien una de las formas de medir la aplicación de un modelo educativo por competencias es el seguimiento de los estudiantes en su proceso de participación en situaciones reales de trabajo, es necesario realizar un seguimiento constante del desenvolvimiento de los mismos, analizar resultados para ir adaptando las mallas curriculares y en especial los contenidos que se impartirán.

En cuanto a los retos sobre la aplicación del modelo, como todo enfoque, aún existen algunas debilidades que deben ir ajustándose durante el proceso de aplicación. Por ejemplo, desarrollar la capacidad de los docentes para impartir metodologías, dinámicas, y didácticas que permitan llevar a la práctica lo que se aprende. Por ello, un aspecto a considerar es 
la de convocar a docentes-especialistas donde los gerentes de hoteles, restaurantes, y agencias de viajes con una larga trayectoria empresarial y profesional ingresan a las aulas para ofrecer su conocimiento.

Finalmente, queda una agenda pendiente del enfoque por competencias el cual para optimizarlo es necesario realizar un análisis permanente de la realidad turística, educativa y laboral, realizar prospectivas de lo que está por venir en la formación de nuevo talento humano para la industria turística.

\section{REFERENCIAS BIBLIOGRÁFICAS}

Argudín, Yolanda (2000). Educación basada en competencias. Recuperado

de http://educacion.jalisco.gob.mx/consulta/educar/19/argudi $\underline{\text { n.html }}$

Banco Mundial. (2010). El mercado laboral peruano durante el auge y la caída. Estudio de país del Banco Mundial. Informe No. 50915PE. LEDEL.

Barrales, Adoración (2012). El enfoque educativo basado en competencias, un reto que enfrenta la Universidad Veracruzana. Revista Educación PUCP. Departamento de Educación PUCP. Recuperado de http://revistas.pucp.edu.pe/index.php/educacion/article/view/ 2884

Comisión de Promoción del Perú para la Exportación y el Turismo [PROMPERU]. (2015). Perfil del Vacacionista Nacional 2014. El Turismo en Cifras. Recuperado de https://issuu.com/visitperu/docs/perfil-del-vacacionistanacional-20?e $=0 / 34112153$

Consejo Nacional de Educación [CNE]. (2005). Hacia un Proyecto Educativo Nacional. Recuperado de http://www.cne.gob.pe/uploads/proyecto-educativonacional/version-pen/hacia-un-proyecto-educativo-nacionalset05.pdf

Dirección General de Investigación y Estudios sobre Turismo y Artesanía [DGIETA]. (2016). Perú: proyección de crecimiento del 
empleo totales en el sector turismo 2015-2021. Recuperado de https://www.mincetur.gob.pe/wp-content/uploads/documentos/turismo/eventos_capacitaciones/III Foro/IIIForo_AP_Estadistica_Seminario/01\%20Seminario\% 20Int_Estudios.pdf

Henley Centre Headlight. (s.f.). Las Tribus Viajeras del Mañana 2020. Recuperado

de http://www.amadeus.com/es/documents/aco/spain/es/Trav ellerTribesWeb.pdf

INEI (2015). Encuesta de ENAHO proyección, metodología actualizada. Le Boterf, Guy (2000). La gestión por competencias. Recuperado de: http://www.guyleboterf-conseil.com/IDEA.PDF

Ministerio de Comercio Exterior y Turismo [MINCETUR]. (2015). Llegada de turistas internacionales 2002-2015. Recuperado de $\quad$ https://www.google.com.pe/?gfe_rd=cr\&ei=0OQV8znDYaw8wfivomwBA\#q=llegada+de+turistas+internaci onales+2002-2015

Ministerio de Comercio Exterior y Turismo [MINCETUR]. (2016). Oferta de establecimientos hoteleros por categoría 2010 y 2016. Recuperado de https://www.mincetur.gob.pe/turismo/normaslegales/prestadores-de-servicios-turisticos/

Ministerio de Trabajo y Promoción del Empleo [MTPE]. (2013). Estudio de la Actividad Turística: Identificación de las ocupaciones demandadas a nivel nacional. Lima: Talleres Gráficos de Corporación Creagrama.

Ministerio de Educación de Colombia (2016). Educación para el trabajo y el desarrollo humano. Recuperado el 3 de setiembre de 2017 de http://www.mineducacion.gov.co/1759/w3-article236469.html

Ministerio de Educación de la República de Corea (s/f). Higher education. Recuperado el 3 de setiembre de 2017 de http://english.moe.go.kr/sub/info.do?m=020105\&s=english

Organización para la Cooperación y el Desarrollo Económicos [OCDE]. (2016). Reporte Avanzando hacia una mejor educación para Perú. Recuperado de https://www.oecd.org/dev/Avanzandohacia-una-mejor-educacion-en-Peru.pdf

Ley General de Turismo. (2009). Recuperado de http://extwprlegs1.fao.org/docs/pdf/per89826.pdf 
Procalidad. (2014). Estudio definitivo del PIP, mejoramiento de la calidad de la educación superior. Lima: Consejo Superior del SINEACE

Regalado, O., y Oré, C, (2009). La educación superior en turismo y las necesidades reales del mercado. Lima: ESAN. Recuperado de http://investigaciones.esan.edu.pe/archivos/DocTrab23.PD $\underline{F}$

Sector hotelero alista más de 100 proyectos hasta el 2018. (2015). El Comercio. Recuperado de http://goo.gl/CNBGle

Superintendencia Nacional de Migraciones. (2018). Recuperado de http://datosturismo.mincetur.gob.pe/appdatosTurismo/Conten t1.html

UNESCO. (1998). La Educación superior en el siglo XXI: Visión y Acción. Declaración mundial sobre Educación Superior. Recuperado de

http://www.unesco.org/education/educprog/wche/declaration _spa.htm 